\title{
Gestión y evaluación participativa de políticas públicas: el caso de los presupuestos participativos ${ }^{1}$
}

Luciano Barbosa de Lima²

\section{Resumen}

Este trabajo tiene por objetivo tratar del tema de la evolución de la gestión en el ámbito de la administración pública para el desarrollo de la práctica de la democracia participativa, tomando como ejemplo el caso del presupuesto participativo, su comienzo en Porto Alegre (Brasil) y los modelos empleados en Europa adaptados a las diferentes realidades. Por fin se discute el tema de la evaluación participativa, sus evoluciones desde el modelo tradicional de evaluación y potencialidades para la mejora de la calidad en la administración pública. Para esto se realiza una breve recopilación sobre la gestión participativa, llevándonos a percibir que los procesos administrativos pasarían por una adaptación natural en las diferentes realidades, así como también su interpretación puede ser diferente. Si la gestión participativa es algo relativamente reciente en términos teóricos, su aplicación y estudio empírico en la administración pública es escaso, en gran parte debido a la falta de apoyo político y limitaciones de recursos económicos y humanos (conocimiento). Entender su importancia y los procesos previstos son fundamentales para emplear cambios en la administración pública y tornarla más eficiente y adaptada al ciudadano.

Palabras clave: Gestión pública. Participación pública. Evaluación de políticas públicas. Presupuesto participativo. Gestión participativa. Evaluación participativa

\footnotetext{
${ }^{1}$ Trabajo presentado para el curso "La calidad de los servicios en las administraciones públicas y su relación con la evaluación de laspolíticas públicas", ministrado por la profesora Dra. Susana Corzo Fernández, en ámbito del Programa de Doctorado en "Economía, Gestión y Control de Entidades y Políticas Públicas” de la Universidad de Granada. 02 de junio de 2008.

${ }^{2}$ Luciano Barbosa de Lima. Doctorando en Economía, Gestión y Control de Entidades y Políticas Públicas por la Universidad de Granada (España), Master (maestría) en Cooperación Internacional y Gestión de Proyectos de Desarrollo por la Universidad de Málaga (España), Licenciado en Relaciones Internacionales por el UniCEUB. Contacto: luciano. balima@gmail.com.
} 


\section{Histórico de la gestión participativa}

Dentro de la evolución de las teorías de la administración, la gestión participativa empieza a tener su origen en los "movimientos de las relaciones humanas"3 difundidas por Elton $\mathrm{Mayo}^{4}$ en la década de 1920 y 1930, un ambiente donde se da hincapié al factor humano en las organizaciones y la racionalidad de la gestión.

En las décadas de 1960 y 1970 surgen movimientos políticos y sociales de trabajadores y organizaciones sindicales reivindicando modalidades más democráticas de gestión, introduciendo formas de cogestión y autogestión.

En la década de 1980 se experimentan varias propuestas de estructuras y procesos de participación, modificando principios y métodos de gestión empresarial, entre ellos propuestas de gestión participativa de Lise Demailly.

Estos cambios de la gestión se deben esencialmente a la importancia que se viene dando a los individuos (su racionalidad, autonomía y estrategias) en las teorías de la administración y del análisis organizacional, y los nuevos "actores organizacionales", sus juegos y dinámicas. También se debe a la influencia de los modelos japoneses de gestión y el concepto de "desarrollo organizacional" y "círculos de calidad" como forma de asociar los trabajadores a la resolución de problemas, la toma de decisiones y comprometimiento en su ejecución.

\section{Gestión participativa en las administraciones públicas y la Democracia participativa}

Las nuevas demandas de la sociedad actual han forzado el estado a "modernizarse" y desarrollar nuevos modelos de gestión que posibiliten un mejor trato de los bienes públicos y sus intereses. Estos modelos de gestión han sido influenciados por los nuevos paradigmas y enfoques de la administración como "la planificación estratégica (Porter); la calidad total (Deming, Duran, Crosby); la reingeniería (Hammer, Champy, Manganelli); la innovación de procesos

\footnotetext{
${ }^{3}$ Traducción del término The human relations movement en: MAYO, Elton. The human relations movement. Disponible en: <http://www.library.hbs.edu/hc/hawthorne/intro. html\#i $>$.

${ }^{4}$ MAYO, Elton. The social problems of an industrialised civilization. [S.l.: s.n.], 1933.
} 
(Davenport), las organizaciones de aprendizaje (Senge), y la reinvención del gobierno (Gaebler y Osborne), entre otros"5, lo que llevó a la incorporación de términos como cliente, calidad, valor, incentivos y flexibilidad a la gestión pública, movimiento conocido también como neoinstitucionalismo.

Actualmente un modelo de gestión participativa de la administración pública es el concepto conocido como Democracia Participativa, mecanismo por el cual la sociedad civil y los actores locales en general no limitan su acción democrática solamente al voto, sino que participan de los procesos de decisión en el ámbito de la administración pública.

\section{El caso del Presupuesto participativo: su nacimiento y evolución en Brasil}

Un buen ejemplo de Democracia Participativa es el Presupuesto Participativo, el cual consiste en "un proceso de deliberación democrática y toma de decisión en el cual los residentes de una ciudad deciden como alocar parte del presupuesto público a través de una serie de asambleas y encuentros a nivel local”, ${ }^{\circ}$ o en otras palabras, práctica por el cual la población local se involucra en el proceso de decisión del uso de parte de los recursos financieros públicos.

Muchos analistas comentan el nacimiento de prácticas de presupuesto participativo en la década de 1970 en el Ayuntamiento de Lages (estado de Santa Catarina), cuando un alcalde buscaba discutir la utilización del presupuesto con la población. En la década de 1980 Brasil pasó por una fase que estudiosos llaman de "participacionista", período en que se valoraba el aspecto participativo en la administración pública, y ganó fuerza en el período dictatorial brasileño.

La primera experiencia del modelo conocido actualmente como Presupuesto Participativo ocurrió en 1989 en Porto Alegre (estado de Rio Grande do Sul) en la gestión del Partido de los Trabajadores (PT) y surgió como resultado de la presión de movimientos populares en participar de decisiones gubernamentales. La

\footnotetext{
${ }^{5}$ RAMÍREZ GUERRA, Carlos Hernán. Gestión participativa y modernización de la gestión pública. Santiago de Chile: [s.n.], 2000. p. 79.

${ }^{6}$ PARTICIPATORY BUDGETING. Home page. Porto Alegre, 2006. Disponible en: <www. participatorybudgeting.org/>. Traducción propia.
} 
diferencia de esta práctica con las anteriores fue que cada ciudadano podía votar sobre cuales tipos de necesidades el gobierno local debería atender en "Plenarias Regionales", atribuyendo mayor peso de voto a aquellas regiones que tenían más carencias de servicios públicos, entre otros criterios.

Algunas otras iniciativas innovadoras fueron implementadas, entre ellas el "Presupuesto Participativo Digital" (votación electrónica por Internet) del Ayuntamiento de Belo Horizonte (estado de Minas Gerais) y el "Presupuesto participativo dirigido a niños (as) y adolescentes" llevado a cabo por los Ayuntamientos de Fortaleza (estado de Ceará) y el Ayuntamiento de São Paulo (estado de São Paulo).

Entre 1997 y 2000, cerca de 140 municipios brasileños iniciaron un proceso de participación con el objetivo de implementar la iniciativa de Presupuesto Participativo. $^{7}$

Reconocida por el Banco Mundial, UN-Habitat (Conferencia de Estambul de 1996) y varios estudiosos internacionales como "Práctica bien sucedida de Gestión Local", esta iniciativa viene siendo diseminada por el mundo, revelando diversas metodologías adaptadas a las diferentes realidades, siendo el modelo de Porto Alegre estructurado por delegados componentes de un Consejo que negocia directamente con los representantes públicos locales la viabilidad de implementación y ejecución de los proyectos y decisiones aprobadas en asamblea.

\section{El presupuesto participativo en Europa}

Con la diversidad de casos y la dificultad de presentar un panorama global, basado en una serie de criterios, el grupo de investigación sobre el presupuesto participativo en Europa ${ }^{8}$ creó un mapa con 6 modelos diferenciables de los casos en este continente: Porto Alegre adoptado para Europa; Participación de intereses organizados; Fondos comunitarios a nivel local y de ciudad; Mesa de negociación

\footnotetext{
${ }^{7}$ TEIXEIRA, Ana Claudia et al. Orçamento participativo: democratização da gestão pública e controle social: as experiências de participação popular na aplicação do dinheiro público municipal. Rio de Janeiro: FASE, 2004.

${ }^{8}$ The research "Participatory Budgets in Europe" has been conducted by the Centre Marc Bloch in Berlin, in cooperation with the Humboldt-University in Berlin and with funds from the Hans-Böckler-Foundation and from the CNRS (France).
} 
Público/Privado; Participación de proximidad; y Asesoramiento en finanzas públicas, como se presenta en el grafico siguiente:

Gráfico 1: Presupuesto participativo en Europa: tipología de procedimientos

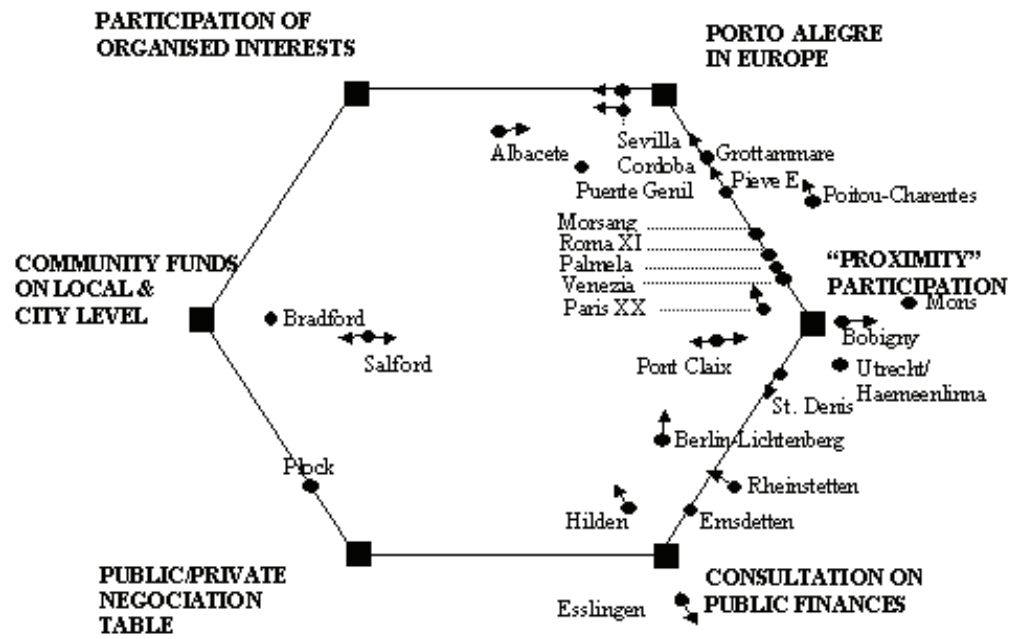

Fuente: SINTOMER, Yves; HERZBERG, Carsten; RÖCKE, Anja. From Porto Alegre to Europe: potentials and limitations of participatory budgeting. Disponible en: $<\mathrm{http} / / / \mathrm{www}$. buergerhaushalt-europa.de/documents/From_Porto_Alegre8.pdf>. p. 6.

En algunos casos el modelo procedimental de Porto Alegre ha sido transformado para adaptarse a otras necesidades. En el modelo "Porto Alegre adoptado para Europa" (Porto Alegre adapted for Europe) se ha traslado el modelo de Porto Alegre en el que el individuo participa en el proceso decisorio y se discuten concretamente inversiones y proyectos, pudiendo ser observado en el caso de la ciudad de Córdoba implementado en 2001; mientras que en el modelo "Participación de intereses organizados" (participation of organised interests), son las federaciones y grupos organizados los principales actores, basado en la lógica neo-corporativista, y tiene en pauta de discusión las líneas políticas a ser seguidos. En ambos procesos se permiten llegar a sugerencias concretas para la resolución de problemas y la clarificación de cuestiones sustanciales. 
Los modelos de "Fondos comunitarios a nivel local y de ciudad" (Community funds on local and city level) y la Mesa de negociación Público/Privado (Public/Private negotiation table) son casos marginados en Europa, presentes en Gran Bretaña y Leste Europeo. Se caracterizan por un espacio donde los recursos son relativamente independientes del presupuesto público local y en el cual el ente público no tiene la última palabra de decisión pero es uno de los actores que aporta recursos financieros, acercándose estos modelos a un fondo de inversiones y presentando distinción cuanto al poder de participación de los actores.

El modelo de Participación de proximidad (Proximity participation) está más presente en Francia y se basa en que los participantes no votan o definen prioridades de proyectos, pero son como "oyentes selectos", teniendo pequeña influencia en el proceso decisorio; y el modelo de Asesoramiento en finanzas públicas (consultation on public finances), que aparece más en Alemania, funciona a partir de una invitación del alcalde a los residentes para debatir propuestas. En estos dos casos la "proximidad" es limitada y se resumen a consultoría, más cercanas a las estratégicas de la Nueva Gestión Pública que las propuestas de Porto Alegre.

\section{La evaluación participativa}

La metodología de evaluación participativa ha empezado con los trabajos de Michael Quinn Patton y David Korten, proponiendo el ejercicio de la evaluación como siendo una experiencia de "aprendizaje organizacional", buscando que los agentes analicen y aprendan de sus propios programas. ${ }^{9}$

En este ámbito identificamos que las principales funciones de seguimiento y evaluación de programas/proyectos son la auditoria para prestación de cuentas y el desarrollo de lecciones aprendidas o aprendizaje organizacional.

\footnotetext{
${ }^{9}$ AUBEL, Judi. Manual de evaluación participativa del programa: involucrando a los participantes del programa en el proceso de evaluación. 2. ed. [S.1]: Catholic Relief Services; Child Survival Technical Support, feb. 2000.
} 
Gráfico 2: Funciones de seguimiento y evaluación de programas

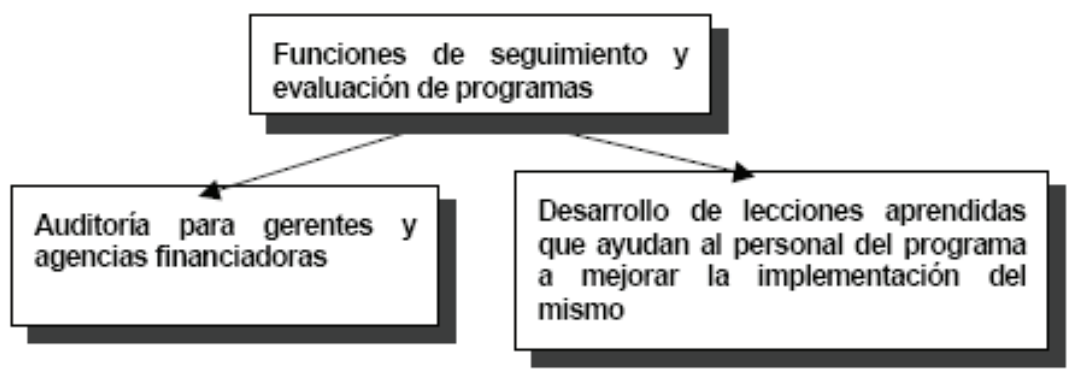

Fuente: AUBEL, Judi. Manual de evaluación participativa del programa: involucrando a los participantes del programa en el proceso de evaluación. 2. ed. [S.l]: Catholic Relief Services; Child Survival Technical Support, feb. 2000. p. 10.

Diversos autores interpretan la evolución que han pasado las evaluaciones en cuatro generaciones ${ }^{10}: 1$ ) evaluaciones ejecutadas desde la perspectiva del donante para justificar sus gastos; 2) evaluaciones centradas en los objetivos, intervenciones con metas prefijadas; 3) no descartando las evoluciones anteriores, la tercera generación de evaluaciones pone énfasis en las interpretaciones y conclusiones de los propios evaluadores; 4) la cuarta generación significa una ruptura con las anteriores y fomenta la participación de los propios beneficiarios en las valoraciones y evaluador para a ser un animador y relator de las discusiones. Esta evolución puede ser descrita como la tabla 1, en la cual podemos identificar algunas de las principales diferencias entre el seguimiento y la evaluación convencionales del seguimiento y la evaluación participativa y comparar su progreso cuanto a objetivos, tratamiento e instrumentos:

${ }^{10}$ ESPAÑA. Metodología de evaluación de la cooperación española. 3. ed. Madrid: Ministerio de Asuntos Exteriores, Secretaría de Estado para la Cooperación Internacional y para Iberoamérica, Oficina de Planificación y Evaluación, 2001. p. 90-91. 
Tabla 1: Principios que distinguen al seguimiento y la evaluación convencionales del seguimiento y la evaluación participativos

\begin{tabular}{|c|c|}
\hline $\begin{array}{l}\text { Seguimiento y evaluación } \\
\text { convencionales }\end{array}$ & $\begin{array}{c}\text { Seguimiento y evaluación } \\
\text { participativos }\end{array}$ \\
\hline $\begin{array}{l}\text { - Tienen por objetivo establecer un crite- } \\
\text { rio acerca del programa a los efectos de } \\
\text { la responsabilidad y no de la potenciaci- } \\
\text { ón de los interesados en el programa. } \\
\text { - Tratan de lograr la objetividad "cientí- } \\
\text { fica" de los hallazgos del seguimiento y } \\
\text { la evaluación, distanciando de ese modo } \\
\text { a los evaluadores externos de los intere- } \\
\text { sados. } \\
\text { - Tienden a hacer hincapié en las nece- } \\
\text { sidades de información de los proveedo- } \\
\text { res de fondos para los programas y los } \\
\text { encargados de formular políticas y no en } \\
\text { las de los ejecutores de los programas ni } \\
\text { las personas afectadas por el programa. } \\
\text { - Se centran en la medición del éxito } \\
\text { alcanzado atendiendo a indicadores } \\
\text { determinados previamente. }\end{array}$ & $\begin{array}{l}\text { - Constituyen un proceso de aprendizaje } \\
\text { individual y colectivo y de fomento de la } \\
\text { capacidad mediante el cual las personas } \\
\text { cobranmayor conciencia de sus puntosfuertes } \\
\text { y débiles, sus realidades sociales más amplias y } \\
\text { susnocionesyapreciacióndelasconsecuencias } \\
\text { del cumplimiento del programa. Este proceso } \\
\text { de aprendizaje crea condiciones que propician } \\
\text { el cambioy la acción. } \\
\text { - Hacen hincapié en diversos niveles de } \\
\text { participación (de bajo a alto) de diferentes } \\
\text { tipos de interesados en el inicio del segui- } \\
\text { miento y la evaluación, la definición de sus } \\
\text { parámetros y su realización. } \\
\text { - Constituyen un proceso social de nego- } \\
\text { ciación entre las diferentes necesidades, } \\
\text { expectativas y cosmovisiones de las per- } \\
\text { sonas. Son un proceso en extremo político } \\
\text { en el que se abordan cuestiones relativas } \\
\text { a la equidad, la autoridad y la transforma- } \\
\text { ción social. } \\
\text { - Consisten en un proceso flexible en cons- } \\
\text { tante evolución y adaptación a las circunstan- } \\
\text { cias y necesidades concretas del programa. }\end{array}$ \\
\hline \multicolumn{2}{|c|}{$\begin{array}{l}\text { Fuente: ESTRELLA, Marisol; GAVENTA, John. Who counts reality? Participatory monitoring and evaluation: a } \\
\text { literature review. IDS Working Paper, n. 70, } 1997 \text { apud UNFPA. Participación de los Interesados en las actividades } \\
\text { de seguimiento y evaluación. [S.I]: Conjunto de Herramientas de Planificación, Seguimiento y Evaluación del } \\
\text { Administrador de Programas, División de Servicios de Supervisión, ago. 2004. Herramienta n. } 4 .\end{array}$} \\
\hline
\end{tabular}

En la tabla 2 hacemos nuevamente una comparación entre la evaluación convencional o también llamado "Plan Riguroso" y la participativa o "Proceso de Aprendizaje", en este caso con base en las diferencias de propósito de la evaluación y la recolección de información: 
Tabla 2: Procedimientos para la evaluación de programas

\begin{tabular}{|l|l|l|}
\hline & \multicolumn{1}{|c|}{ "Plan Riguroso" } & "Proceso de Aprendizaje" \\
\hline Propósito & $\begin{array}{l}\text { - Medir el progreso del } \\
\text { plan de trabajo }\end{array}$ & $\begin{array}{l}\text { - Medir los } \\
\text { logros cuantitativos } \\
-\quad \text { Desarrollar lecciones } \\
\text { aprendidas }\end{array}$ \\
\hline $\begin{array}{l}\text { Recolección } \\
\text { de información }\end{array}$ & $\begin{array}{l}\text { limitado a las metas del } \\
\text { plan }\end{array}$ & $\begin{array}{l}\text { y más amplio } \\
- \text { Dimensiones } \\
\text { y preguntas adicionales }\end{array}$ \\
\hline $\begin{array}{l}\text { Métodos de recolección } \\
\text { de información }\end{array}$ & - Cuantitativo \\
- Análisis objetivo & $\begin{array}{l}\text { y Cuantitativo } \\
\text { - Juicio "subjetivo" }\end{array}$ \\
\hline $\begin{array}{l}\text { Responsabilidad } \\
\text { de recolección } \\
\text { de información }\end{array}$ & $\begin{array}{l}\text { - Evaluadores externos } \\
\text { Fuente: AUBEL, Judi. Manual de evaluación participativa del programa: involucrando a los } \\
\text { participantes del programa en el proceso de evaluación. 2. ed. [S.I]: Catholic Relief Services; } \\
\text { Child Survival Technical Support, feb. 2000. p. 13. }\end{array}$ & $\begin{array}{l}\text { - Participantes } \\
\text { del programa (gerentes y } \\
\text { ejecutores) }\end{array}$ \\
\hline
\end{tabular}

En el procedimiento participativo a nivel organización, el coordinador de la evaluación trabaja con los participantes del programa en todas las etapas de su trabajo, siendo los participantes estos los responsables de los resultados del programa. ${ }^{11}$ En un nivel de proyectos de desarrollo podrían participar varios interesados, como la comunidad cuya situación el programa pretende cambiar, el personal sobre el terreno del proyecto que realiza las actividades, los administradores de programas que supervisan la implementación del programa, los proveedores de fondos y otros encargados de tomar decisiones que deciden la línea de acción en relación con el programa, y los partidarios y los detractores del programa, así como otros interesados que influyen en el entorno del programa. ${ }^{12}$ La participación es muy importante para la mejora de la

${ }^{11}$ ESPAÑA. Metodología de evaluación de la cooperación española. 3. ed. Madrid: Ministerio de Asuntos Exteriores, Secretaría de Estado para la Cooperación Internacional y para Iberoamérica, Oficina de Planificación y Evaluación, 2001. p. 90-91.

${ }^{12}$ Davies, C. T., 1998 apud UNFPA. Participación de los Interesados en las actividades de seguimiento y evaluación. [S.1]: Conjunto de Herramientas de Planificación, Seguimiento y Evaluación del Administrador de Programas, División de Servicios de Supervisión, ago. 2004. Herramienta n. 4. 
calidad, teniendo en cuenta que la información estaría compartida para una gama más amplia de actores y las políticas empleadas podrían estar en más cercanas y aceptadas por aquellos que, al final de la actividad, serían los evaluadores-beneficiarios.

\section{La evaluación participativa dentro del ciclo de proyecto}

Dentro del Ciclo de Proyectos y teniendo en cuenta sus nuevos propósitos, podríamos conectar la Evaluación Participativa no sólo a la etapa de Evaluación y Auditoria como el caso convencional, sino que también y sustancialmente a la de implementación, momento en el cual se puede identificar los procesos y las lecciones aprendidas de estos procesos.

Gráfico 3: El Ciclo de Proyectos y la Evaluación Participativa

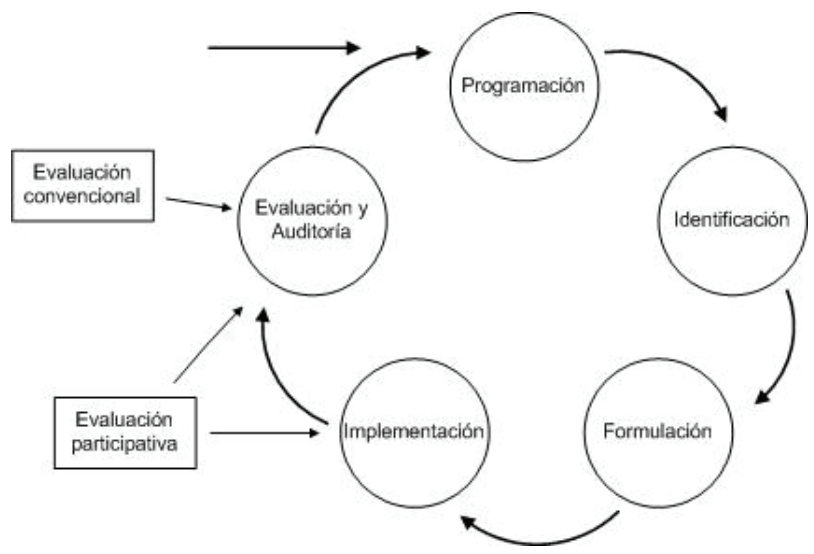

Producción propia, adaptado al Ciclo de Proyecto propuesto por la Comisión Europea en el documento Project Cycle Management Guidelines, 2004.

\section{Etapas de la Evaluación Participativa: planificación y realización}

Aube ${ }^{13}$ sistematiza las fases de la planificación y realización de la metodología de la evaluación participativa de la siguiente forma:

13 AUBEL, Judi. Manual de evaluación participativa del programa: involucrando a los participantes del programa en el proceso de evaluación. 2. ed. [S.1]: Catholic Relief Services; Child Survival Technical Support, feb. 2000. 
Fases de la Metodología de Evaluación Participativa

- Fase I: Reuniones de pre-planificación

- Paso 1: Definición de metas y objetivos de la evaluación

- Paso 2: Identificación de los miembros del equipo de evaluación (participantes)

- Paso 3: Planificación de los aspectos logísticos y administrativos

- Paso 4: Desarrollo del marco de trabajo visual del proyecto

- Paso 5: Orientación a los facilitadores del taller de planificación de la evaluación

- Fase II: Taller de planificación de la evaluación

- Paso 6: Organización de los participantes en torno a un grupo de trabajo

- Paso 7: Definición de los aspectos de la evaluación

- Paso 8: Identificación de fuentes y técnicas de recolección de información

- Paso 9: Desarrollo de instrumentos para la recolección de información

- Paso 10: Conclusión de la elaboración de la muestra de lugares para la recolección de información y de personas a encuestar

- Fase III: Trabajo de campo: Preparación para el trabajo de campo, recolección de información y análisis de datos

- Paso 11: Preparación de los equipos del trabajo de campo: Técnicas de recolección de información y logística

- Paso 12: Realización de entrevistas y observaciones

- Paso 13: Análisis de la información recolectada

- Paso 14: Resumen de los hallazgos del trabajo de campo

- Fase IV: Taller para la formulación de lecciones aprendidas

- Paso 15: Formulación de lecciones aprendidas para cada pregunta de la evaluación

- Paso 16: Análisis del proceso de evaluación por parte del equipo

- Fase V: Resumen de los resultados de 1 a evaluación

- Paso 17: Resumen de los hallazgos y lecciones aprendidas de la evaluación

- Fase VI: Desarrollo del plan de acción

- Paso 18: Desarrollo de un plan de acción en base a los hallazgos de la evaluación

- Fase VII: Finalización y distribución del informe de la evaluación

- Paso 19: Redacción del informe de evaluación

- Paso 20: Distribución y discusión de los resultados de la evaluación con personas que colaboran con el programa

Estas etapas son construidas para ser un mero instrumento de planificación de la implementación de una evaluación participativa, sin embargo su éxito no está asegurado con el simple cumplimiento de estas fases, sino que tiene relación con 
diversos otros factores políticos, sociales, económicos e institucionales. Entre los más importantes aspectos para una efectiva evaluación es el apoyo organizacional de los directivos de la institución (gobierno en el caso de políticas públicas) para la formación de un equipo coordinador de la evaluación y la coordinación de un facilitador que defina las técnicas y estimule la participación.

Además, es importante tomar en cuenta los principios propuestos por Aube ${ }^{14}$ para este tipo de evaluación: creatividad, responsabilidad, transparencia, consideración de la administración como un todo responsable de los efectos de ese todo, innovación y la igualdad a través del libre acceso a la información pública, y formación del ciudadano.

\section{Conclusiones}

Teniendo en cuenta las crecientes demandas, complejidades y expectativas cuanto a las políticas públicas, la gestión participativa y específicamente el presupuesto participativo y la evaluación participativa pueden ser piezas importantes para la solución de controversias, descentralización de competencias y de toma de decisiones, favoreciendo mejor implementación de políticas que posibilitan el Establecimiento de las Prioridades, Concentración de las Energías, Aumento de la Objetividad, Sistematización de los objetivos para el territorio, Colaboración entre los sectores público y privado, Creación de una cultura estratégica común, Refuerzo del liderazgo de las autoridades territoriales, Identificación más clara del uso más efectivo de los recursos y fondos públicos, y así Facilidad para conseguir más recursos públicos y privados. ${ }^{15}$ Estos criterios podrían ser causa y efecto de una participación que promovería un incremento de la calidad del servicio público.

Para ello es necesario que esta nueva gestión se constituya en un cambio organizacional respaldado por los directivos, con perspectiva de largo plazo y sistemas organizacionales reforzados por técnicas que induzcan y

\footnotetext{
${ }^{14}$ AUBEL, Judi. Manual de evaluación participativa del programa: involucrando a los participantes del programa en el proceso de evaluación. 2. ed. [S.1]: Catholic Relief Services; Child Survival Technical Support, feb. 2000.

${ }^{15}$ Adaptación del texto GARCÍA LIZANA, Antonio et al. Planificación estratégica sostenible de redes territorial: teoría y práctica. Málaga: Universidad de Málaga/Debates, 2005.
} 
maximicen las aportaciones del recurso humano a la mejora de productividad, al proceso de decisión y otras actividades necesarias para el desarrollo de las políticas. ${ }^{16}$

Además, las técnicas y metodologías pueden tener diferentes formas y modelos, adecuados a cada realidad y sus necesidades. Para eso es importante un análisis minucioso del contexto al cual se trabaja para entender como se dirigen las cosas, de que forma y que modelo puede ser mejor aplicado.

\section{Participatory management and evaluation of public policies: the case of the participatory budgeting}

\section{Abstract}

This paper aims to discuss the issue of the evolution of management in the field of public administration for the development of participatory democracy practices, taking as example the case of participatory budgeting, his beginning in Porto Alegre (Brazil) and the models used in Europe adapted to different realities. Finally it discusses the issue of participatory evaluation, its evolution from the traditional model of assessment and potential for quality improvement in public administration. For this, a brief compilation on participatory management has been done, leading us to perceive that the administrative process would go through a natural adaptation to the different realities, as well as your interpretation may be different. If participatory management is a relatively recent development in theoretical terms, its implementation and empirical study in public administration is scant, largely due to the lack of political support and limited financial and human resources. Understand its importance and fundamental processes are expected to promote changes in public administration and make it more efficient and adapted for citizens

Keywords: Public management. Public participation. Policy evaluation. Participatory budgeting. Participatory management, Participatory evaluation.

\footnotetext{
${ }^{16}$ BELCHER, Johny, 1996, apud RAMÍREZ GUERRA, Carlos Hernán. Gestión participativa y modernización de la gestión pública. Santiago de Chile: [s.n.], 2000.
} 


\section{Referencias}

AUBEL, Judi. Manual de evaluación participativa del programa: involucrando a los participantes del programa en el proceso de evaluación. 2. ed. [S.l]: Catholic Relief Services; Child Survival Technical Support, feb. 2000.

BARTLE, Phil. Participación en la revisión anual: involucrar al personal en la supervisión, valoración y planificación. Disponible en: $<\mathrm{http}: / / w w w . s c n . o r g / m p f c /$ modules/pm-ars.htm>.

ESPAÑA. Metodología de evaluación de la cooperación española. 3. ed. Madrid: Ministerio de Asuntos Exteriores, Secretaría de Estado para la Cooperación Internacional y para Iberoamérica, Oficina de Planificación y Evaluación, 2001.

GARCÍA LIZANA, Antonio et al. Planificación estratégica sostenible de redes territorial: teoría y práctica. Málaga: Universidad de Málaga/Debates, 2005.

MAYO, Elton. The human relations movement. Disponible en: $<$ http://www.library. hbs.edu/hc/hawthorne/intro.html\#i >.

MAYO, Elton. The social problems of an industrialised civilization. [S.l.: s.n.], 1933.

PARTICIPATORY BUDGETING. Home page. Porto Alegre, 2006. Disponible en: $<$ www.participatorybudgeting.org/>.

RAMÍREZ GUERRA, Carlos Hernán. Gestión participativa y modernización de la gestión pública. Santiago de Chile: [s.n.], 2000.

SINTOMER, Yves; HERZBERG, Carsten; RÖCKE, Anja. From Porto Alegre to Europe: potentials and limitations of participatory budgeting. Disponible en: $<$ http://www.buergerhaushalt-europa.de/documents/From_Porto_Alegre8.pdf $>$.

TEIXEIRA, Ana Claudia et al. Orçamento participativo: democratização da gestão pública e controle social: as experiências de participação popular na aplicação do dinheiro público municipal. Rio de Janeiro: FASE, 2004.

UNFPA. Participación de los Interesados en las actividades de seguimiento y evaluación. [S.I]: Conjunto de Herramientas de Planificación, Seguimiento y Evaluación del Administrador de Programas, División de Servicios de Supervisión, ago. 2004. Herramienta n. 4.

WAMPLER, Brian. A guide to participatory budgeting. [S.l.: s.n.], 2000. 